\title{
Iterative learning control for stroke rehabilitation with input dependent muscle fatigue modeling
}

\author{
Fons Luijten, Bing Chu and Eric Rogers
}

\begin{abstract}
The consequences of a stroke is a major and increasing problem world wide. Many people who suffer a stroke are left with permanent impairment but the possibility exists that suitable rehabilitation could increase mobility and, for example, enable independent living. This, in turn, requires effective rehabilitation where it is known that currently available methods are relatively poor and are not well suited to home use, where the latter aspect is critical to improving practice and reducing costs. An accepted method to relearn lost function, such as reaching out to an object, is repeated attempts with learning from previous from those already completed with the application of applied stimulation if required. This requirement is analogous to iterative learning control and much progress, with supporting clinical trials data, has been reported on using this engineering design method to regulate the applied stimulation such that patient improvement in completing the task corresponds to increasing voluntary input and reduced stimulation. The applied stimulation in this application can induce muscle fatigue and this paper gives new result on enhancing the control laws to mitigate this unwanted effect.
\end{abstract}

\section{INTRODUCTION}

Annually, 15 million people world-wide suffer a stroke and up to a third of these are left with permanent impairment. Other demographic patterns and, in particular, aging populations place even more strain on the resources for patient care and rehabilitation. Stroke is an age-related disease [1] and all of these factors contribute to an increasing burden on long-term health and related resources. Hence there is a pressing need to improve the effectiveness of treatments to achieve independence.

A common cause of a stroke is blockage of a blood vessel in the brain, where as a result regions downstream are starved of blood. Consequently, the connecting nerve cells die and this usually leads to partial paralysis on one side of the body, termed hemiplegia. The brain cells that die as a result of a stroke cannot regrow but new connections can be made using the brain's spare capacity. In particular, the brain is continually and rapidly changing and as new skills are learned, new connections are formed and redundant ones disappear. Relearning skills after a stroke is the same process as a person learning an everyday task, such as reaching out to a cup, and requires sensory feedback during repeated practice

\footnotetext{
This work was undertaken when the first author was on an Erasmus exchange at the Department of Electronics and Computer Science, University of Southampton, UK.

Fons Luijten is with the Department of Mechanical Engineering, Eindhoven University of Technology

Bing Chu and Eric Rogers are with the Department of Electronics and Computer Science, University of Southampton, Southampton SO17 1BJ, UK b.chu (etar) decs.soton.ac.uk
}

of a task. This requires movement skills but the affects of the stroke means that these are almost always very poor and hence feedback on performance is not obtained.

Stroke survivors commonly have a complex pattern of upper limb motor impairments with a loss in functional abilities such as reaching. The coupling between reaching and independence is reflected in measures of function independence, including the Bartel index [2] where the ability to reach is essential for approximately $50 \%$ of activities that make up daily living tasks. Currently, the level of upper limb recovery following a stroke is poor and it has been reported [3] that complete recovery occurs in less than $15 \%$ of patients with initial paralysis. This and the age-related factor are among the major reasons why there is a critical need to improve the effectiveness of treatments. If the stage were reached where rehabilitation could be moved outside the hospital, which requires mobile technology, then reduced costs could result.

The literature on conventional therapy plus motor learning theory, e.g., [4], provides evidence that functional recovery can be achieved through the facilitation of motor control and skill acquisition and restoration of muscle power through repetitive resistance exercises [5], in addition to the variety of tasks and feedback. This knowledge has motivated the development of novel treatments, such as robot-aided therapy, which could provide the basis longer-term for a translation of rehabilitation clinics from labor-intensive work to technology-assisted operations and also an opportunity for repetitive movement practice. Reviews of the robotic therapy literature, available in [6], [7] and the cited references. For the upper limb suggest that robot-assisted treatment improves motor control of the proximal upper limb and may improve functional outcomes.

Rehabilitation robots are power driven or mechanically supported devices that assist a patient with limited physical capability to undertake repetitive exercises. The resulting sensory feedback is known to be associated with cortical changes that facilitate the recovery of functional movement. Functional electrical stimulation (FES) has been found to be applicable as another method in promoting cortical connectivity to enable recovery, which is motivated by a growing body of clinical evidence and theoretical support from neurophysiology and motor learning research, again see [6], [7] for references to the literature and [8] for an overview of FES with a control systems perspective.

Application of FES to a muscle causes electrical impulses to travel along the nerves in the same way as electrical impulses from the brain and if the stimulation is carefully 
regulated a useful movement can be made. In stroke rehabilitation FES is applied in combination with the patients voluntary effort with the aim of a specific recovery of voluntary power. A wide range of algorithms have been applied to the control of FES for both the upper and lower limbs, where again the literature is covered in [6], [7] and the cited references. In recent work Iterative Learning Control (ILC), see the references cited in [6], [7] has been applied to regulate the FES applied in robotic-assisted upper limb stroke rehabilitation.

This research started with a planar daily living motivated task, reaching out over a table top, where the patient was asked to track a supplied reference trajectory whilst attached to a robotic arm with assistive FES applied to the relevant muscle, i.e., the triceps. During each attempt, the error between the desired trajectory and that produced by the patient was measured, the arm reset to the starting location and in the time before the next attempt an ILC law was used to compute the FES to be applied. This work proceeded to a clinical trial where the required property that as the patient improves with repeated attempts voluntary effort increased and the level of FES required decreased [6], [7] was detected. This application area for ILC has been extended to 3D tasks, such as reaching and extending the forearm, where there is a need to stimulate more than one muscle and again supporting clinical trial results are available [6], [9], [7].

In application, FES applied to muscles is at a higher frequency and is hence a contributory factor to muscle fatigue. If the muscle suffers from fatigue then the force output drops and the treatment session has to stop to allow recovery, which almost certainly means the session must end and the patient return at another time, see [8] for a detailed control systems/modeling discussion of this area. The previous research on ILC for upper-limb stroke rehabilitation did not explicitly account for muscle fatigue in the model used for control law design but this aspect must be addressed if the use of model based control laws in this and related problem areas is to proceed.

One approach to overcoming, or at least reducing, the effects of fatigue was considered in [10], which introduced a representation for the effects of fatigue into the model for the response of the muscle to applied FES with a compensating feedback loop around the model used. The results of a detailed simulation based evaluation of the new design is given where the dynamic model is constructed from data collected from patients participating in a previous clinical study of this ILC application. Such an evaluation is an essential step before seeking ethical approval for patientbased trials. This paper gives new results based on an enhanced muscle model and gives a performance between no compensation for muscle fatigue, the previous work and the new design.

\section{BACKGROUND}

The same setup as in [10] is considered and starts with Figure 1, which consists of the human arm supported by a mechanical rig. This rig can measure the position of the arm and contains springs to counteract gravity. In Figure 1 a) the combined system is shown, Figure $1 \mathrm{~b}$ ) shows the Structure of the mechanical support with all Degrees Of Freedom (DOF) and Figure $1 \mathrm{c}$ ) shows the DOF of the human arm.

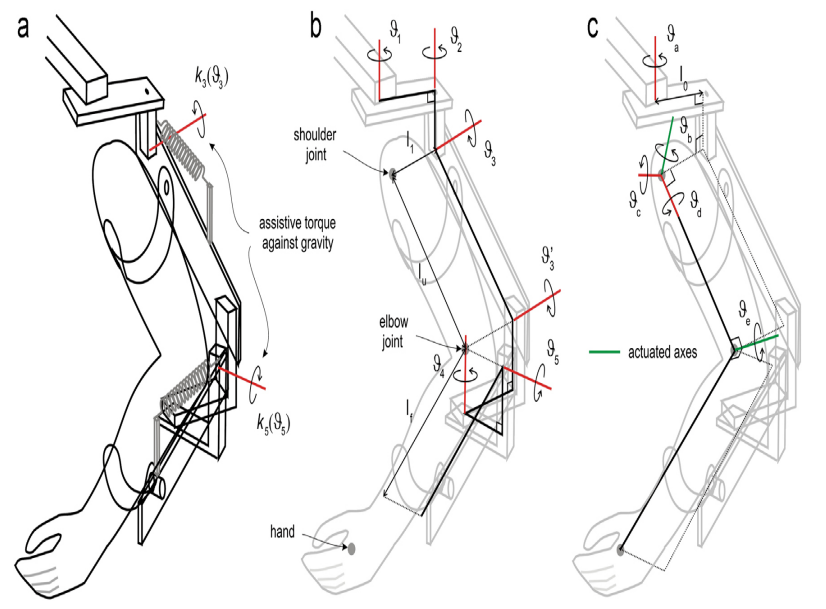

Fig. 1. System setup and corresponding DOF; a) Combined system, b) Mechanical support, c) Human arm. (Source: [10])

The position of the human arm can be described by $\Phi=$ $\left[\begin{array}{lllll}\vartheta_{a} & \vartheta_{b} & \vartheta_{c} & \vartheta_{d} & \vartheta_{e}\end{array}\right]^{\top}$. Using a Lagrangian approach, the differential equations describing the dynamics of the support and arm can be described as

$$
B(\Phi) \ddot{\Phi}+C(\Phi, \dot{\Phi}) \dot{\Phi}+F(\Phi, \dot{\Phi})+G(\Phi)+K(\Phi)=\tau(u, \Phi, \dot{\Phi}),
$$

in which $B(\Phi)$ represents the inertial matrix, $C(\Phi, \dot{\Phi})$ the Coriolis matrix and $F(\Phi, \dot{\Phi})$ the vector of non-conservative forces acting on the system. The term $G(\Phi)$ contains moments from gravity acting on the system and $K(\Phi)$ is the vector of moments from the springs designed to counteract gravity. An extended description of these terms can be found in [7]. The input

$$
\tau(u, \Phi, \dot{\Phi})=\left[\begin{array}{lllll}
0 & \tau_{b}\left(u_{b}, \Phi_{b}, \dot{\Phi}_{b}\right) & 0 & 0 & \tau_{e}\left(u_{e}, \Phi_{e}, \dot{\Phi}_{e}\right)
\end{array}\right]^{\top}
$$

is created by the stimulated muscle. The task for the patient considered in [10] is that of lifting the affected arm and then reaching out from the elbow. It is well known that stroke patients experience great difficulty in lifting the affected arm and hence part of this robot compensates for gravity. The muscles involved are the triceps $\left.u_{b}(t)\right)$ and the anterior deltoid $\left(u_{e}(t)\right)$ and complete details of the robot configuration, how the target is presented and the supporting software development can again be found in [6], [9], [7] (and the relevant cited references). Hence only $\vartheta_{b}$ and $\vartheta_{e}$ are the axes in that are actuated by means of electrical stimulation. The torques in this vector $\tau(u, \Phi, \dot{\Phi})$ are created as described next.

The relationship between the torques $\tau_{b}$ and $\tau_{e}$ and the applied FES is referred to as the muscle model. Many models are available to describe the muscle dynamics. A comparison of some of these models is given in [8]. In this paper, the model consists of the non-linear activation dynamics which 
are modelled using a Hammerstein structure. The model can be split up in two parts, the linear activation dynamics $h_{L A D}$ and a static non-linearity $h_{I R C}\left(u_{i}\right)$. The non-linearity $h_{I R C}\left(u_{i}\right)$ describes the Isometric Recruitment Curve (IRC), which maps the stimulation input $u_{i}$ to the steady-state torque $T_{m, i}$ and is given by

$$
h_{I R C, i}\left(u_{i}\right): T_{m, i}=c_{1, i}\left|\frac{e^{c_{2, i} u_{i}}-1}{e^{c_{2, i} u_{i}}+c_{3, i}}\right|, \quad i=\{b, e\} .
$$

In this equation $c_{1, i}, c_{2, i}$ and $c_{3, i}$ are parameters specific for each muscle and can be experimentally determined, see, e.g., [11], [12] and the relevant cited references in these papers.. The linear activation dynamics $h_{L A D}$ describe the relation between $T_{m, i}$ and the fatigue free torque $\tau_{m, i}$. These dynamics are modelled by a second-order critically damped linear system given by the state-space model

$$
\begin{aligned}
\dot{x}_{i} & =\left[\begin{array}{cc}
0 & 1 \\
-\omega_{n}^{2} & -2 \omega_{n}
\end{array}\right] x_{i}+\left[\begin{array}{l}
0 \\
1
\end{array}\right] h_{I R C, i}\left(u_{i}\right) \\
\tau_{m, i} & =\left[\begin{array}{ll}
\omega_{n}^{2} & 0
\end{array}\right] x_{i}, \quad i=\{b, e\},
\end{aligned}
$$

where $\omega_{n}$ is the natural frequency and $x_{i}=\left[\begin{array}{ll}x_{i, 1} & x_{i, 2}\end{array}\right]^{\top}$ is the state for muscle $i$. The muscle model is used to simulate the non-fatigued torque $\tau_{m, i}$ and the modeling representation of fatigue used in this paper is discussed next.

A muscle is in general subjected to fatigue. The torque provided by the muscle will decrease over time when the applied FES is constant. Previous research [10] used a time dependent and iteration dependent fatigue model $f(t, k)$ given by $\tau_{i}=\left(1-k_{f} t\right) \lambda^{k-1} \tau_{m, i}$ in which $k_{f}$ and $\lambda$ are constants determining the time and iteration fatigue rates respectively. The model implies that the muscle will fatigue during a trial even if no input is applied and the arm does not move, which is counter-intuitive. A more intuitive model is used in [13], which proposes a dynamic model describing fatigue as well as recovery from fatigue as a function of input. This model is given by

$$
\begin{aligned}
\tau_{i} & =\phi_{i} \tau_{m, i} \\
\dot{\phi}_{i} & =\frac{1}{T_{f a t, i}}\left(\phi_{\text {min }, i}-\phi_{i}\right) \bar{\tau}_{m, i}+\frac{1}{T_{r e c, i}}\left(1-\phi_{i}\right)\left(1-\bar{\tau}_{m, i}\right), \\
\bar{\tau}_{m, i} & =\left|\frac{1}{\tau_{m, i_{\max }}} \tau_{m, i}\right| \quad i=\{b, e\}
\end{aligned}
$$

in which $\phi_{\min , i} \in[0,1]$ is the minimum fatigue constant indicating the minimum level of fatigue the muscle can reach. $\phi_{i} \in\left[\phi_{m i n, i}, 1\right]$ is the fatigue factor and $T_{f a t, i}$ and $T_{r e c, i}$ are the time constants for fatigue and recovery respectively, which need to be estimated based on measurements. $\bar{\tau}_{m, i} \in$ $[0,1]$ is the normalized positive input. Note that if no fatigue is present in the system, it holds that $\phi_{i}=1$. Between two trials, the system is reset to its initial condition during which the new ILC input is calculated and the muscle has a chance to recover from fatigue. The resting time between trials is denoted as $t_{r e c}$, during which the muscle model will be simulated with $\bar{\tau}_{m, i}=0$ as input.
The control design is split up in two parts. First the feedback controllers are designed consisting of a slave controller and a master controller. Next the ILC design is discussed. The structure of the control design is given in Figure 2. The subscript $k$ serves as an indicator for the trial number for ILC purposes and is left out for the feedback design. It is assumed that the angles $\vartheta_{b}$ and $\vartheta_{e}$ of the human arm can be measured as well as the fatigued torques $\tau_{b}$ and $\tau_{e}$. All other DOF's are not accounted for in the control design.

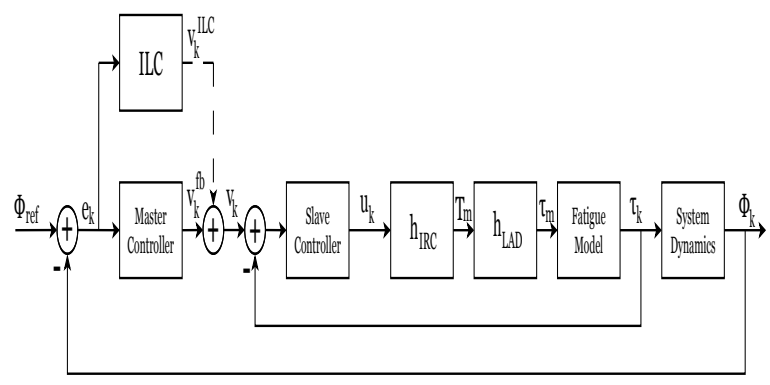

Fig. 2. Control structure of the rehabilitation system.

\section{CONTROL LAW DESIGN}

The slave controller is designed to achieve fast tracking of the applied input and to deal with fatigue present in the system. In the previous research [10] the fatigue is considered as an unknown disturbance on the muscle model and a linearising controller is applied disregarding any fatigue. The non-linearity of the muscle $h_{I R C}$ is assumed to be monotonic and known. Implementing the inverse of $h_{I R C}$ in the slave controller will result in the linearising feedback law

$$
u=\left[\begin{array}{l}
u_{b} \\
u_{e}
\end{array}\right]=\left[\begin{array}{l}
h_{I R C, b}^{-1}\left(\omega_{n}^{2} x_{b, 1}+2 \omega_{n} x_{b, 2}+\frac{v_{b}^{\prime}}{\omega_{n}^{2}}\right) \\
h_{I R C, e}^{-1}\left(\omega_{n}^{2} x_{e, 1}+2 \omega_{n} x_{e, 2}+\frac{v_{e}^{\prime}}{\omega_{n}^{2}}\right)
\end{array}\right],
$$

in which $v_{b}^{\prime}$ and $v_{e}^{\prime}$ are the new inputs to be designed. Applying this linearising controller results in the transferfunction matrix $P_{\text {muscle }}(s)$ and, using $v_{i}^{\prime}=C_{i}^{s}\left(v_{i}-\tau_{i}\right)$, the closed-loop transfer-function matrix $G_{\text {muscle }}(s)$ given by

$$
\begin{aligned}
P_{\text {muscle }}(s) & =\left[\begin{array}{cc}
\frac{\phi_{b}}{s^{2}} & 0 \\
0 & \frac{\phi_{e}}{s^{2}}
\end{array}\right], \\
G_{\text {muscle }}(s) & =\left[\begin{array}{cc}
\frac{C_{b}^{s} \phi_{b}}{s^{2}+C_{b}^{s} \phi_{b}} & 0 \\
0 & \frac{C_{e}^{s} \phi_{e}}{s^{2}+C_{e}^{s} \phi_{e}}
\end{array}\right],
\end{aligned}
$$

in which $C_{i}^{s}$ is the slave controller chosen as a proportional gain, $C_{i}^{s}=K_{p_{i}}^{s}$. The input to this transfer-function matrix is $v=\left[\begin{array}{ll}v_{b} & v_{e}\end{array}\right]^{\top}$. Also if the patient at any instant reaches full fatigue $\left(\phi_{i}=0\right)$ the treatment session cannot continue. Next the master controller is designed as a proportional gain plus derivative controller such that the input $v$ is given by

$$
\begin{aligned}
v & =v^{f b}+v^{I L C} \\
& =\left[\begin{array}{l}
K_{p_{b}}^{m}\left(\vartheta_{b, r e f}-\vartheta_{b}\right)+K_{d_{b}}^{m}\left(\dot{\vartheta}_{b, r e f}-\dot{\vartheta}_{b}\right)+v_{b}^{I L C} \\
K_{p_{e}}^{m}\left(\vartheta_{e, r e f}-\vartheta_{e}\right)+K_{d_{e}}^{m}\left(\dot{\vartheta}_{e, r e f}-\dot{\vartheta}_{e}\right)+v_{e}^{I L C}
\end{array}\right](8)
\end{aligned}
$$


where $v^{I L C}$ is the feedforward calculated by the ILC controller discussed next.

In this paper the ILC controller is designed Newton-based ILC, see, e.g. [14]. The Newton method is chosen for the ILC design but alternatives are possible. First the overall system is rewritten as a function of the ILC input $v^{I L C}$, which includes the controlled slave and master loops. The result is

$$
\begin{aligned}
& \dot{x}=f\left(x, v^{I L C}\right) \\
& y=h(x)=\left[\begin{array}{ll}
\vartheta_{b} & \vartheta_{e}
\end{array}\right]^{\top}
\end{aligned}
$$

In this paper two cases are considered. Case (1) disregards any fatigue present in the system in the ILC design and is designed using

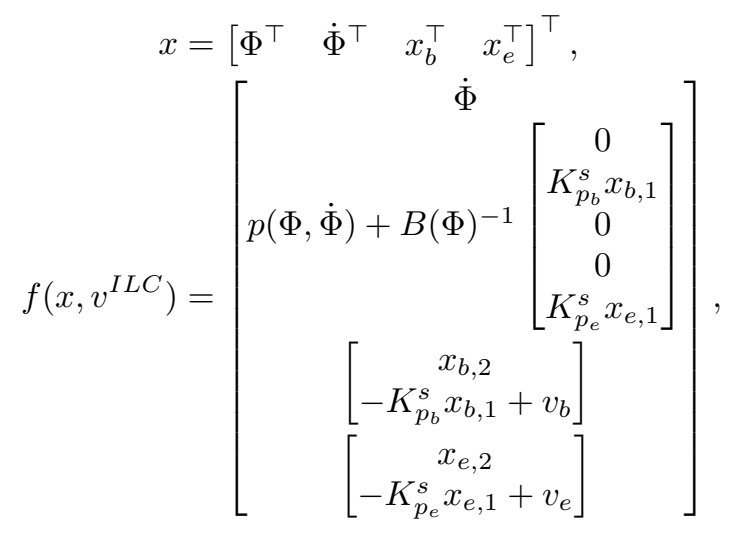

with $p(\Phi, \dot{\Phi})=-B(\Phi)^{-1}(C(\Phi, \dot{\Phi}) \dot{\Phi}+F(\Phi, \dot{\Phi})+G(\Phi)+$ $K(\Phi))$ and where the inputs $v_{b}$ and $v_{e}$ are given in 8. Case (2) includes the fatigue model in the ILC design, starting from

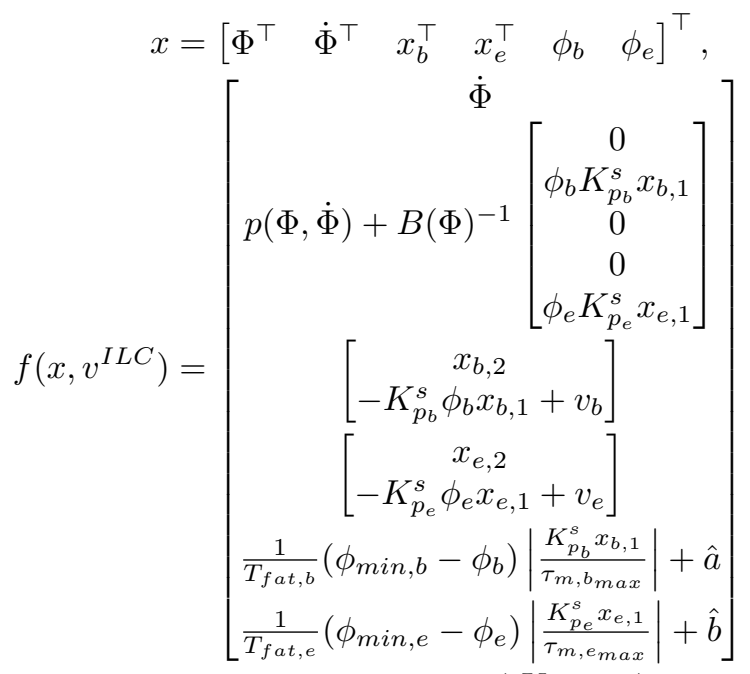

$$
\text { with } \hat{a}=\frac{1}{T_{r e c, b}}\left(1-\phi_{b}\right)\left(1-\left|\frac{K_{p_{b}}^{s} x_{b, 1}}{\tau_{m, b_{\max }}}\right|\right)
$$

$$
\text { and } \hat{b}=\frac{1}{T_{r e c, e}}\left(1-\phi_{e}\right)\left(1-\left|\frac{K_{p_{e}}^{s} x_{e, 1}}{\tau_{m, e_{\max }}}\right|\right) \text {. }
$$

In order to calculate the ILC inputs for trial $k+1$, the system in (9) is first sampled with sampling time $T_{s}$ to obtain the discrete time state space model

$$
\begin{aligned}
\boldsymbol{x}_{\boldsymbol{k}}(n+1) & =\boldsymbol{f}\left(\boldsymbol{x}_{\boldsymbol{k}}(n), \boldsymbol{v}_{\boldsymbol{k}}^{\boldsymbol{I} \boldsymbol{L C}}(n)\right) \\
\boldsymbol{y}_{\boldsymbol{k}}(n) & =\boldsymbol{h}\left(\boldsymbol{x}_{\boldsymbol{k}}(n)\right),
\end{aligned}
$$

with the sample number $n \in[0,1, \ldots, N]$ in which $N=$ $T_{\text {end }} / T_{s}$. All bold symbols are the discrete time equivalents of their continuous-time counterparts. The system starts each trial from the same initial condition $\boldsymbol{x}_{\boldsymbol{k}}(0)=\boldsymbol{x}_{0}$ and can therefore be written as

$$
\boldsymbol{y}_{\boldsymbol{k}}(n)=\boldsymbol{g}\left(\boldsymbol{v}_{\boldsymbol{k}}^{\boldsymbol{I} \boldsymbol{L C}}(n)\right) .
$$

Using the Newton method, the ILC input for the next trial is given by

$$
\begin{aligned}
v_{k+1}^{I L C} & =v_{k}^{I L C}+z_{k+1} \\
z_{k+1} & =g^{\prime}\left(v_{k}^{I L C}\right)^{-1} e_{k},
\end{aligned}
$$

where $\boldsymbol{e}_{\boldsymbol{k}}=\Phi_{r e f}-\boldsymbol{y}_{\boldsymbol{k}}$ is the tracking error and $\boldsymbol{g}^{\prime}\left(\boldsymbol{v}_{\boldsymbol{k}}^{\boldsymbol{I} \boldsymbol{L} \boldsymbol{C}}\right)$ is equivalent to the linearization of $g\left(\boldsymbol{v}_{\boldsymbol{k}}^{I L C}\right)$ around $\boldsymbol{v}_{\boldsymbol{k}}^{I L C}$. This linearization results in the linear time-varying system

$$
\begin{aligned}
\tilde{\boldsymbol{x}}(n+1) & =A(n) \tilde{\boldsymbol{x}}(n)+B(n) \tilde{\boldsymbol{u}}(n) \\
\tilde{\boldsymbol{y}}(n) & =C(n) \tilde{\boldsymbol{x}}(n) \\
& \text { with } \\
A(n) & =\left.\frac{\partial \boldsymbol{f}}{\partial \boldsymbol{x}_{\boldsymbol{k}}}\right|_{\boldsymbol{v}_{\boldsymbol{k}}^{I L C}(n), \boldsymbol{x}_{\boldsymbol{k}}(n)}, \\
B(n) & =\left.\frac{\partial \boldsymbol{f}}{\partial \boldsymbol{v}_{\boldsymbol{k}}^{\boldsymbol{I} \boldsymbol{L C}}}\right|_{\boldsymbol{v}_{\boldsymbol{k}}^{I L C}(n), \boldsymbol{x}_{\boldsymbol{k}}(n)} \\
C(n) & =\left.\frac{\partial \boldsymbol{h}}{\partial \boldsymbol{x}_{\boldsymbol{k}}}\right|_{\boldsymbol{v}_{\boldsymbol{k}}^{I L C}(n), \boldsymbol{x}_{\boldsymbol{k}}(n)},
\end{aligned}
$$

in which $\tilde{\boldsymbol{u}}=\boldsymbol{z}_{\boldsymbol{k}+\boldsymbol{1}}$ and $\tilde{\boldsymbol{y}}$ needs to track $\boldsymbol{e}_{\boldsymbol{k}}$. Solving this system using a second ILC loop will result in the input $z_{k+1}$ that will regulate the trial error dynamics. For this loop, Norm Optimal ILC (NOILC) [15] is used, where the subscript $j$ is used to indicate the iteration number. On iteration $j$ the input and output to the system are $\tilde{\boldsymbol{u}}_{j}$ and $\tilde{\boldsymbol{y}}_{j}$ respectively. Using NOILC the next input $\tilde{\boldsymbol{u}}_{j+1}$ is calculated by minimizing the cost function

$$
\begin{aligned}
J\left(\tilde{\boldsymbol{u}}_{j+1}\right) & =\sum_{n=0}^{N-1}\left(\left(\boldsymbol{e}_{\boldsymbol{k}}-\tilde{\boldsymbol{y}}_{j}\right)^{\top} Q\left(\boldsymbol{e}_{\boldsymbol{k}}-\tilde{\boldsymbol{y}}_{j}\right)\right. \\
& \left.+\left(\tilde{\boldsymbol{u}}_{j+1}-\tilde{\boldsymbol{u}}_{j}\right)^{\top} R\left(\tilde{\boldsymbol{u}}_{j+1}-\tilde{\boldsymbol{u}}_{j}\right)\right),
\end{aligned}
$$

in which $Q \in \mathbb{R}^{+}$and $R \in \mathbb{R}^{+}$are symmetric weighting matrices. When $j=10$ or when the error $\boldsymbol{e}_{\boldsymbol{k}}-\tilde{\boldsymbol{y}}_{j}$ is sufficiently small, the ILC loop is stopped and the resulting $\tilde{\boldsymbol{u}}_{j}=z_{k+1}$ is used to calculate the new ILC input for the Newton method using (14).

\section{Performance evaluation}

The performance of the two designs has been compared in simulation using a system model built from stroke patient data using estimates for the fatigue model parameters. First case (1) is evaluated, in which no compensation for fatigue is implemented. The 2-norm of the error does not converge 
in this case which is caused by the fatigue varying too much from trial-to-trial without compensation. Allowing for a longer recovery time between successive trials such that the muscle will recover more from the fatigue is the only option if fatigue arises is the only way achieving error convergence in this case. However, with stroke patients the required ethical approval will specify a maximum time that a session can last. In this time, all required measurements necessary to construct the model must be made and also the task explained and demonstrated to the patient. Moreover, failure to complete a session for any reason can have a demotivating for some patients.

For case (2) the 2-norm of the error converges as seen in Figure 3.
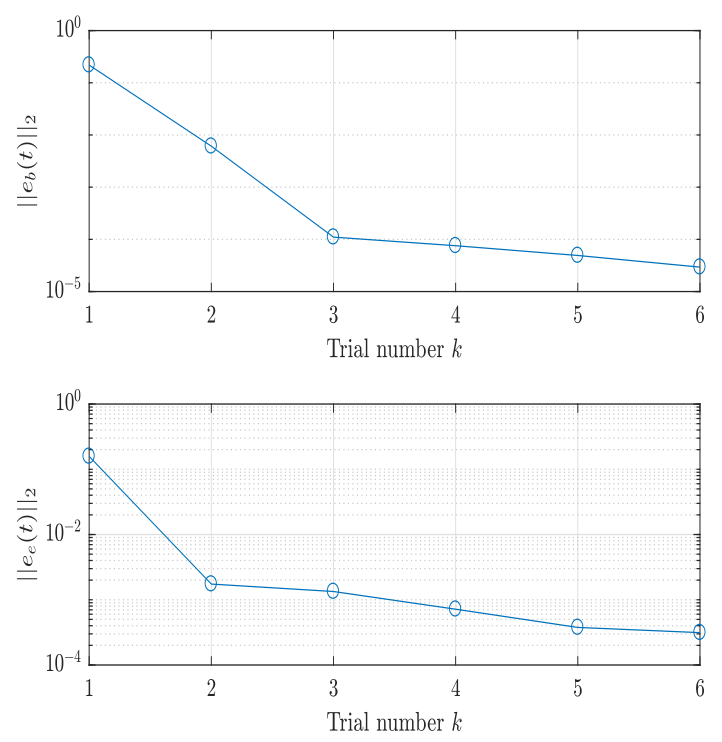

Fig. 3. 2-norm of the error for Newton based ILC in case (2).

In this case the fatigue is taken into account in the ILC loop. Because the ILC loop is constructed model based, the fatigue model is assumed to be known. Since in the first trial no ILC is applied and fatigue is not compensated for in the slave controller, the closed loop system has to be stable under influence of the fatigue. The assumption is made here that the muscle is not fatigued at the start of the ILC trials. Note that the next ILC input is calculated from a linearization around the previous measured/estimated state $x$. Since the fatigue on the next trial is different from the previous trial, this method will only work for small changes in fatigue between trials. Multiple simulations confirm this result, however more change is allowed than in case (1). The error is shown in Figure 4 for trials $k=1,2$ and 5 .

This plot exhibits some oscillations in the error signal of $e_{e}$ near the end of the trial after applying the ILC law. These oscillations were also observed for case (1) and are expected to be introduced into the system by solving the linear system in the NOILC problem. More research into the origin of these oscillations can still be done. Figure 5 shows the input
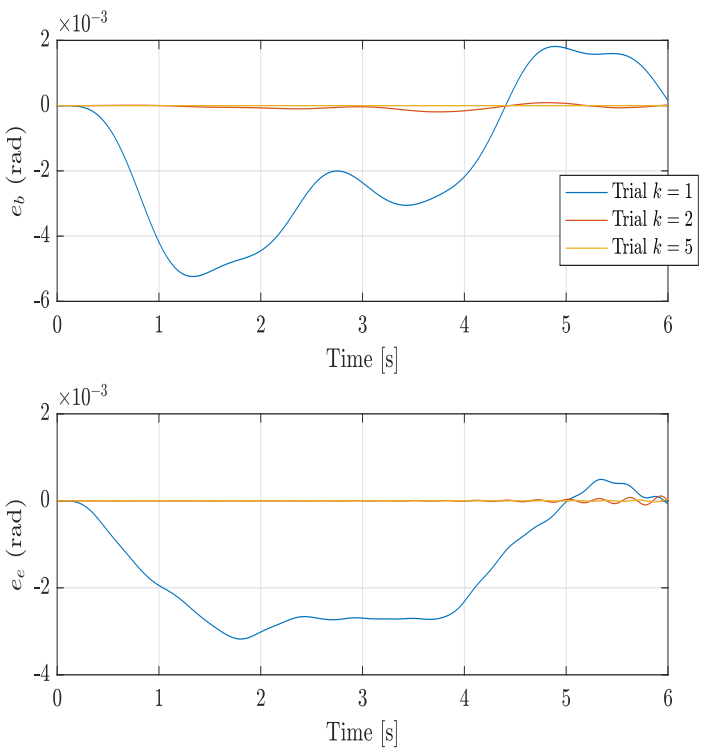

Fig. 4. The error for Newton based ILC in case (2) for trials $k=1,2,5$.

to the system at trial $k=6$. The input satisfies bounds for clinical trial approval, but is larger than in case (1). This was expected since the fatigue is now compensated for in the input. The results in this part are superior when compared with those in [10].
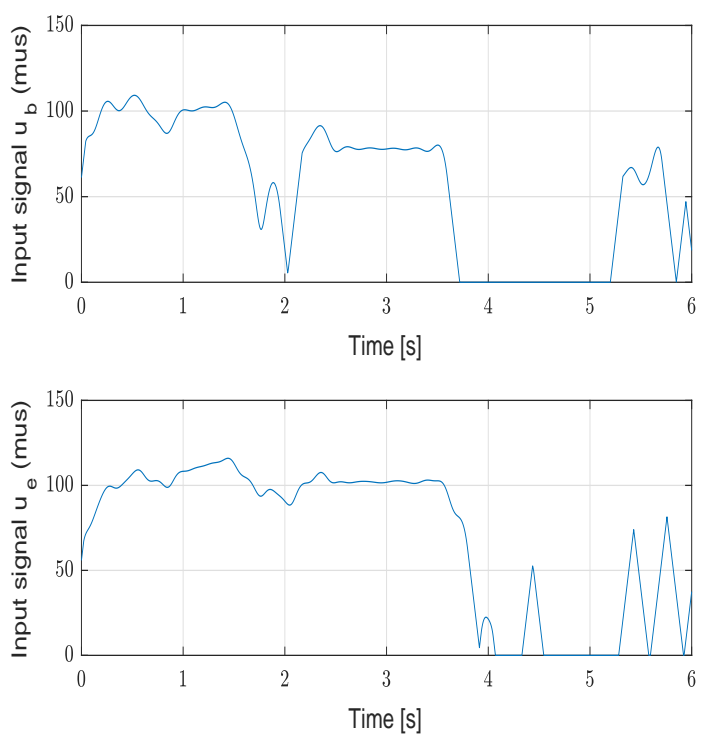

Fig. 5. The input for Newton based ILC in case (2).

\section{Conclusions}

Previous researhc has established that ILC can be used to regulate the level of FES applied to the muscles of patients undergoing robotic-assisted upper limb stroke rehabilitation, where the patient makes repeated attempts at a prescribed finite duration task with FES applied to the relevant muscles. 
Once an attempt, or trial is complete, the patients arm is returned to the starting location and in this time, plus a rest period, an ILC law uses the error measured on the previous trial is used in updating the FES to be applied on the next trial. If the patient is improving with each successive attempt then the level of FES required should decrease and the voluntary effort supplied by the patient increase.

This paper has continued the development of another critical factor not considered in the research, including clinical trials [6], [9], [7] i.e., the effects of muscle fatigue that can arise for a number of reasons and, in particular, since the applied FES is at higher frequency. This problem was considered in [10] but using a very simplified representation for fatigue. This paper has used a more representative model for muscle fatigue and the benefits have been mirrored in the simulation results given. Further development, involving more in depth simulations and the use of other control configurations is required prior to making an application for ethical approval to undertake clinical trials. The need to overcome fatigue in other applications of ILC in rehabilitation/asistive technology is also pressing, see, as two examples, [16], [17].

\section{REFERENCES}

[1] National Audit Office, Reducing brain damage: faster access to better stroke care (2004) Available at http://www.nao.org.uk/publications/0506/reducing brain damage.aspx.

[2] J. J. M. F. van der Putten, J. C. Hobart, J. A. Freeman, A. J. Thompson, Measuring change in disability after inpatient rehabilitation: comparison of the responsiveness of the barthel index and the functional independence measure, Journal of Neurology, Neurosurgery \& Psychiatry 66 (1999) 480-484.

[3] H. T. Hendricks, A. C. van Limbeek, J., C. Geurts, A, M. J. Zwarts, Motor recovery after stroke: a systematic review of the literature (2002).

[4] J. R. De Kroon, M. J. Ijzerman, J. J. Chae, G. J. Lankhorst, G. Zilvold, Relation between stimulation characteristics and clinical outcome in studies using electrical stimulation to improve motor control of the upper extremity in stroke, Journal of Rehabilitation Medicine 37.
[5] H. I. Krebs, J. J. Palazzolo, L. Dipietro, M. Ferraro, J. Krol, K. Rannekleiv, B. T. Volpe, N. Hogan, Rehabilitation robotics: performancebased progressive robotic-assisted therapy, Autonomous Robots 15 (1) (2003) 7-20.

[6] C. T. Freeman, E. Rogers, A.-M. Hughes, J. H. Burridge, K. L. Meadmore, Iterative learning control in healthcare: electrical stimulation and robotic-assisted upper limb stroke rehabilitation, IEEE Control Systems Magazine 32 (1).

[7] C. T. Freeman, E. Rogers, J. H. Burridge, A.-M. Hughes, K. L. Meadmore, Iterative learning control for electrical stimulation and stroke rehabilitation, Control, Automation and Robotics, SpringerVerlag, London, UK, 2015.

[8] C. L. Lynch, M. R. Popovic, Functional electrical stimulation: closedloop control of induced muscle contractions, IEEE Control Systems Magazine 28 (2008) 40-50.

[9] K. L. Meadmore, A.-M. Hughes, C. T. Freeman, Z. Cai, D. Tong, J. H. Burridge, E. Rogers, Function electrical stimulation mediated by iterative learning control and $3 \mathrm{~d}$ robotics reduces motor impairment in chronic stroke, Journal of Neuroengineering and Rehabilitation (2012) doi: 10.1186/1743-0003-9-32.

[10] W. Xu, B. Chu, E. Rogers, Iterative learning control for roboticassisted upper limb stroke rehabilitaiton in the presence of muscle fatigue, Control Engineering Practice 31 (2014) 63-72.

[11] F. Le, I. Markovsky, C. T. Freeman, E. Rogers, Identification of electrically stimulated muscle models of stroke patients, Control Engineering Practice 18 (4) (2010) 87-97.

[12] F. Le, I. Markovsky, C. T. Freeman, E. Rogers, Recursive identification of Hammerstein systems with application to electrically stimulated muscle, Control Engineering Practice 20 (4) (2012) 386-396.

[13] R. Riener, J. Quintern, G. Schmidt, Biomechanical model of the human knee evaluated by muscular stimulation, Journal of Biomechanics 29 (9) (1996) 1157-1167.

[14] T. Lin, D. H. Owens, J. Hatonen, Newton-mehtod based iterative learning control for discrete nlnlinear systems, International Journal of Control 79 (10).

[15] N. Amann, D. H. Owens, E. Rogers, Iterative learning control for discrete time systems with exponential rate of convergence, Proceedings of the Institution of Electrical Engineers: Control Theory and Applications 143 (1996) 217-224.

[16] T. Seel, T. Schauer, J. Raisch, Monotonic convergence of iiterative learning control systems with variable pass length, International Journal of Control (2016a) DOI: 10.1080/00207179.2016.1183172.

[17] T. Seel, C. J. Werner, J. Raisch, T. Schauer, Iterative learning control of a drop foot neuroprosthesis-generating physiological foot motion in paretic gait by automatic feedback control, Control Engineering Practice 48 (2016b) 87-97. 